We present a case of a 60-yearold male patient with diagnosis of non-Hodgkin's lymphoma (NHL): diffuse large B cell lymphoma (non-Hodgkin's DLBCL), who achieved complete remission after first-line treatment. Eleven months later he was admitted to our hospital again with symptoms of the disease's systemic relapse and central nervous system (CNS) infiltration. He developed new and serious symptoms of complete pituitary gland insufficiency. Magnetic resonance imaging findings and hormone serum levels confirmed the CNS infiltration and insufficiencies of the pituitary as well as its dependent peripheral glands. Chemotherapy and hormonal substitution significantly improved the patient's condition for several months. That is why, in the case of deteriorated patient condition, we have to consider possible secondary development of pathological processes in the course of the primary disease.

Key words: pituitary insufficiency, diabetes insipidus, central nervous system infiltration, lymphoma.

Contemp Oncol (Pozn) 2014; 18 special issue DOI: $10.5114 /$ wo.2014.40597

\section{Insufficiency of the pituitary gland resulting from central nervous system infiltration in course of non-Hodgkin's DLBCL lymphoma recurrence}

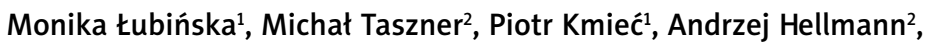 \\ Krzysztof Sworczak ${ }^{1}$
}

${ }^{1}$ Department of Endocrinology and Internal Diseases, Medical University of Gdansk, Poland

${ }^{2}$ Department of Haematology and Transplantology, Medical University of Gdansk, Poland

\section{Introduction}

Central nervous system infiltration by lymphoproliferative neoplasms, such as non-Hodgkin lymphomas, may take place as a primary process when the tumour's mass is found solely in this location, or secondarily, i.e. featuring an extranodular site of a generalised non-Hodgkin's lymphoma (NHL).

Primary CNS lymphomas are rare diseases with poor prognosis, necessitating radically aggressive treatment [1].

Secondary CNS lymphomas (SCNSL) are defined as NHL present both in and outside of the CNS. Secondary CNS lymphomas may infiltrate meninges, deep brain structures, spinal cord and cerebro-spinal fluid (CSF) [2]. The lesions may be single, diffused or disseminated [3].

Secondary CNS involvement is rarely found on NHL diagnosis (with a case frequency median of 1.1\%); more often, patients with $\mathrm{NHL}$ recurrence after treatment are affected [4]. A proposed reason for this is the insufficient CNS penetration of $1^{\text {st }}$-line drugs [5].

Several factors have been identified in assessing the risk of NHL recurrence with CNS involvement that point at clinical situations in which the implementation of prophylactic treatment should be considered. Until now, research has not resulted in a single accepted treatment scheme for SCNSL; thus, treatment often remains individualised.

Hypophysis insufficiency $(\mathrm{HI})$ as a result of neoplasmatic growth is a rare condition. Among primary tumours that yield metastases to the gland, breast and lung cancers are most frequent, and less commonly gastrointestinal tract carcinomas and malignant melanomas are enumerated [6]. Thus far, only a few cases of a primary hypophysis involvement by a primary or secondary NHL have been reported [7-10].

Depending on the time frame and the extent of hypophysis infiltration, as well as the patient's age, a typical constellation of symptoms of gland insufficiency develop. These may be accompanied by symptoms resulting from hypothalamus and/or optic chiasm involvement [11, 12].

Endocrine insufficiency symptoms presented by a patient with NHL-infiltrated pituitary may be mistakenly considered as being caused by the lymphoma itself or due to the chemotherapy applied against it.

In this article we would like to stress the importance of considering symptoms not only as a consequence of the primary disease or the side-effects of its treatment, but also as symptoms of a secondarily developing pathological process in its course. Attentive patient supervision facilitates the diag- 
nosis of new events triggered by the disease and allows to undertake the correct additional treatment.

\section{Case report}

A 59-year-old man was referred to a day ward of the Haematology and Transplantology Clinic of the Medical University of Gdansk hospital due to fatigue (WHO performance status scale score was 2), considerable weight loss, generalised lymphadenopathy and splenomegaly.

Laboratory findings revealed anaemia (haemoglobin value of $8.4 \mathrm{~g} / \mathrm{dl}$ ) and increased lactate dehydrogenase (LDH) level (1089 IU/I). A DLBCL CD20+, CD3- non-Hodgkin lymphoma with a Ki67 index value of $50 \%$ was diagnosed in a lymph node histological examination. IIIS B clinical stage of the lymphoma was stated using a computed tomography scan.

Based on the present risk factors of the International Prognostic Index (IPI), i.e. 3, the patient was assigned to the high-medium risk group.

Six cycles of first-line treatment were applied - R-CHOP (rituximab, cyclophosphamide, doxorubicin, vincristine, prednisone). A complete remission was achieved, as confirmed in a PET scan.

Eleven months after the treatment in a follow-up PET scan, recurrence of the NHL was suggested, which was confirmed the following month when a SCNSL was also suspected. On admission to the Haematology Clinic the patient complained of headaches, vertigo, imbalance and nausea. These, along with extreme fatigue, were initially recognised as symptoms resulting from general condition worsening due to the progress of the disease.

Secondary peripheral endocrine gland insufficiency symptoms dominated in the clinical presentation, i.e. con-

Table 1. Results of hormonal, biochemical and osmolal analyses in a patient with pituitary lymphoma infiltration before hormonal substitution

\begin{tabular}{|c|c|}
\hline Tests and normal ranges & Value \\
\hline TSH (N: 0.34-4.94 mIU/l) & 0.06 \\
\hline fT4 (N: 9.01-19.05 pmol/l) & 8.93 \\
\hline fT3 (N: 2.63-5.7 pmol/l) & 2.88 \\
\hline ACTH $(\mathrm{N}:<46 \mathrm{pg} / \mathrm{ml})$ & $<10$ \\
\hline Cortisol 8.00 (N: 116-1060 nmol/l) & 28.7 \\
\hline Cortisol 20.00 (N: 47-458 nmol/l) & 61.4 \\
\hline FSH (N: 1.3-13.5 mlU/ml) & 0.41 \\
\hline LH (N: $1.8-8.16 \mathrm{U} / \mathrm{l})$ & $<0.7$ \\
\hline Testosterone (N: 5.76-28.14 nmol/l) & 0.43 \\
\hline Serum osmolality (N: 270-295 mOsm/l) & 301 \\
\hline Urine osmolality (N: 250-1300 mOsm/l) & 186 \\
\hline $\mathrm{Na}(\mathrm{N}: 135-145 \mathrm{mEq} / \mathrm{l})$ & 152 \\
\hline $\mathrm{K}(\mathrm{N}: 3.5-5.1 \mathrm{mEq} / \mathrm{l})$ & 4.2 \\
\hline Urine specific gravity (1.018-1.030 kg/l) & 1.001 \\
\hline
\end{tabular}

siderable weakness with muscle adynamism (the patient was unable to walk on his own), apathy, sleepiness, nausea with anorexia, thirstiness, periodically polyuria and hypotonia. Type B symptoms were absent.

On neurological examination a slight dropping of the left angle of the mouth, tongue pointing toward the left side, abdominal hypoaesthesia and hyporeflexia of the right side, and a lack of the plantar reflex of the left foot were seen.

Cerebro-spinal fluid examination showed normal cell count and no substantial biochemical alterations. A CT scan confirmed the involvement of structures above the diaphragm. Bone marrow was not infiltrated, and LDH concentration and CBC values were normal.

Central nervous system MRI showed the infiltration of hypophysis' infundibular stem, optic chiasm and lower hypothalamus surface. These results, along with clinical signs, suggested CNS involvement with the pituitary. In conclusion, stage IV NHL was confirmed.

Hormonal tests confirmed complete hypophysis insufficiency, i.e. decreased hypophysis and peripheral hormone concentrations. Imbalance of electrolytes, serum and urine osmolality confirmed a lack of vasopressin - diabetes insipidus (Table 1). Other laboratory results showed hyperlipidaemia and slight anaemia.

The patient was treated according to a reversed CODOX-M scheme (cyclophosphamide, vincristine, doxorubicin and methotrexate, with the last drug given initially highly-dosed, and the CODOX part ten days thereafter), intrathecal doses of methotrexate, cytosine arabinoside and prednisone, as well as hormonal substitution. After two chemotherapy cycles a remission was achieved without hypophysis insufficiency symptoms, and the patient's general condition improved greatly.

A follow-up MRI showed no infiltrate of the hypophysis-hypothalamic region. However, the patient still required hormonal substitution.

In light of weak chemotherapy tolerance and the attainment of complete remission no further chemotherapeutic treatment was undertaken and adjuvant CNS teleradiotherapy with a total of 36 Gy dose in 18 fractions was applied.

Two months later another NHL progression was stated. In a CT scan, stage IV of the disease was revealed with bone marrow, liver and spleen involvement. A neurologically depressed consciousness level was seen - yet again suggesting CNS infiltration. Life-saving chemotherapy was attempted but was abandoned because of septic complications and general condition worsening. The patient died with multiple organ failure symptoms.

\section{Discussion}

Central nervous system infiltration in the course of $\mathrm{NHL}$ is a rare event and can be used to be estimated 5 to $10 \%$ of cases [2, 13, 14]; however, there are new reports.

In 2010 the Polish Lymphoma Research Group published detailed guidelines on diagnostic, prophylactic and therapeutic procedures regarding CNS involvement of diffuse large B cell lymphomas [15]. 
Boehme et al. analysed 1222 elderly patients with aggressive B-cell lymphoma (mostly DLBCL), treated with courses of CHOP or R-CHOP. The number of patients who developed CNS disease was fifty-eight, which constituted $4.8 \%$ of the analysed patients [16].

In recently published papers by Schmitz et al., the authors analysed 2210 younger patients with aggressive B-cell lymphoma treated in various studies for the incidence and risk factors of CNS infiltration [17]. Fifty-six of those patients (2.6\%) developed CNS disease. According to their conclusions, patients with higher age-adjusted International Prognostic Index (aalPI 2 or 3) showed higher risk (4.2-9.7\%) and no significant reduction of CNS disease with rituximab, while patients with aalPI 0 or 1 showed a low risk for CNS disease (0-0.5\%) and rituximab decreased the risk. Another conclusion was that CNS prophylaxis with intrathecal methotrexate was of no significant benefit.

Central nervous system disease is characterised by a very poor prognosis [2]. Certain risk factors on NHL diagnosis may suggest prophylactic treatment against CNS involvement by applying intrathecal or high-dose systemic chemotherapy [18]. These factors include advanced stage of the disease, two or more extranodal sites involved, patient age less than 60, increased serum LDH concentration, presence of B-symptoms, involvement of bone marrow, testicles, spinal column, paranasal sinuses, orbits, breasts or retroperitoneal lymph nodes, decreased albumin concentration or some specific histopathological subtypes of NHL [15-20]. Some of the above risk factors were present in our patient, and the application of prophylactic treatment penetrating to the CNS may have been beneficial.

Typical NHL symptoms such as weakness, fatigue, night sweats, fever and weight loss in case of CNS involvement can be even more intense. Most frequent CNS-infiltration related symptoms are cephalgia, seizures, plegias and paralyses, paresthesias as well as psychiatric disorders and quantitative and qualitative consciousness disturbances [2, 3]. Furthermore, adverse chemo- and radiotherapy effects may mask symptoms due to the decline of neoplasm-infiltrated structures, which may then lead astray the attention of the physician.

Diagnosed relatively rarely, metastases to the hypophysis usually affect adults and elderly patients. Clinically most frequently reported are diabetes insipidus resulting from hypothalamus or posterior hypophysis lobe involvement, hyperprolactinaemia due to infundibular stem damage or visual field defect caused by optic chiasm involvement, and much less commonly posterior and anterior hypophysis lobe disorders [7, 21].

Primary CNS lymphomas and secondary CNS infiltration by NHLS occur rarely. Symptoms resulting from hypophysis function impairment - particularly unspecific symptoms such as headaches, weakness, weight loss, apathy and nausea - may be associated with the disease itself or explained as a consequence of intensive treatment while their true cause remains unnoticed. This in turn largely delays the implementation of hormonal substitution and reduces the therapeutic efficacy as a whole $[9,10$,
21]. Consequently, CNS infiltrations are often found only in post-mortem examinations.

\section{Conclusions}

The case reported here illustrates the necessity of a wide perspective when considering a patient with a neoplasmatic disease. Accepting the most straightforward explanation of the observed symptoms without an in-depth analysis of all data may lead to a delay of serious but treatable complications.

Disease progression was observed in our patient with CNS infiltration, and he developed complete bi-lobular hypophysis insufficiency symptoms.

Adequate diagnosis enabled application of hormonal substitution treatment that prevented life-threatening endocrine crises (adrenal, thyroid). The patient was able to enjoy a satisfactory quality of life and continue haematological treatment.

The issue of CNS prophylaxis of patients with aggressive B-cell lymphoma, when the identified risk factors mentioned above are present, is still difficult and requires further research and precise guidelines.

\section{Authors declare no conflict of interest.}

\section{References}

1. Lister A, Abrey LE, Sandlund JT. Central nervous system lymphoma. Hematology Am Soc Hematol Educ Program 2002; 283-296.

2. Hollender A, Kvaloy S, Lote K, Nome O, Holte H. Prognostic factors in 140 adult patients with non-Hodgkin's lymphoma with systemic central nervous system (CNS) involvement. A single centre analysis. Eur J Cancer 2000; 36: 1762-1768.

3. Yoshida S, Morii K, Watanabe M, Saito T. Characteristic features of malignant lymphoma with central nervous system involvement. Surg Neurol 2000; 53: 163-167.

4. Gleissner B, Chamberlain M. Treatment of CNS dissemination in systemic lymphoma. J Neurooncol 2007; 84: 107-117.

5. Mazhar D, Stebbing J, Bower M. Non-Hodgkin's lymphoma and CNS: prophylaxis and therapy in immunocompetent and HIV-positive individuals. Expert Rev Anticancer Ther 2006; 6: 335-341.

6. Kunert-Radek J. Choroby podwzgórza i przysadki. W: Choroby wewnętrzne. Szczeklik A (red.). Medycyna Praktyczna, Kraków 2005; 1012.

7. Liu JK, Sayama C, Chin SS, Couldwell WT. Extranodal NK/T-cell lymphoma presenting as a pituitary mass. Case report and review of the literature. J Neurosurg 2007; 107: 660-665.

8. Pekic S, Milicevic S, Colovic N, Colovic M, Popovic V. Intravascular large B-cell lymphoma as a cause of hypopituitarism: gradual and late reversal of hypopituitarism after long-term remission of lymphoma with immunochemotherapy. Endocrine 2008; 34: 11-16.

9. Megan Ogilvie C, Payne S, Evanson J, Lister TA, Grossman AB. Lymphoma metastasizing to the pituitary: an unusual presentation of a treatable disease. Pituitary 2005; 8: 139-146.

10. Partap S, Spence AM. Spontaneously relapsing and remitting primary CNS lymphoma in an immunocompetent 45-year-old man. J Neurooncol 2006; 80: 305-307.

11. Lee MT, Lee TI, Won JG, Chau WK, Yang HJ, Li JC, Lin HD, Tang KT. Primary hypothalamic lymphoma with panhypopituitarism presenting as stiff-man syndrome. Am J Med Sci 2004; 328: 124-128.

12. Bolanowski M, Kuliszkiewicz-Janus M, Sokolska V. Diffuse malignant lymphoma type B with optic chiasm infiltration, visual disturbances, hypopituitarism, hyperprolactinaemia and diabetes insipidus. Case report and literature review. Endocrinol Pol 2006; 57: 642-647. 
13. Levitt LJ, Dawson DM, Rosenthal DS, Moloney WC. CNS involvement in the non-Hodgkin's lymphomas. Cancer 1980; 45: 545-552.

14. Lee KW, Yi J, Choi IS, et al. Risk factors for poor treatment outcome and central nervous system relapse in diffuse large B-cell lymphoma with bone marrow involvement. Ann Hematol 2009; 88: 829838.

15. Giebel S, Walewski J, Krawczyk-Kuliś M, et al. Profilaktyka i leczenie zajęcia ośrodkowego układu nerwowego w nowotworach układu chłonnego. Hematologia 2010; 1: 352-358.

16. Boehme V, Schmitz N, Zeynalova S, Loeffler M, Pfreundschuh M. CNS events in elderly patients with aggressive lymphoma treated with modern chemotherapy (CHOP-14) with or without rituximab: an analysis of patients treated in the RICOVER-60 trial of the German High-Grade Non-Hodgkin Lymphoma Study Group (DSHNHL). Blood 2009; 113: 3896-3902.

17. Schmitz N, Zeynalova S, Glass B, et al. CNS disease in younger patients with aggressive B-cell lymphoma - an analysis of patients treated on the Mabthera International Trial and trials of the German High-Grade Non-Hodgkin Lymphoma Study Group. Ann Oncol 2012; 23: 1267-1273.

18. Tomita N, Kodama F, Kanamori H, Motomura S, Ishigatsubo Y. Prophylactic intrathecal methotrexate and hydrocortisone reduces central nervous system recurrence and improves survival in ag gressive on-Hodgkin lymphoma. Cancer 2002; 95: 576-580.

19. Kisiel E, Wosztyl A. Pierwotne i wtórne chłoniaki centralnego układu nerwowego. Wspolczesna Onkol 2004; 8: 136-141.

20. Joks M, Myśliwiec K, Lewandowski K. Primary breast lymphoma - review of the literature and report of three cases. Arch Med Sci 2011; 7: 27-33.

21. Komninos J, Vlassopoulou V, Protopapa D, Korfias S, Kontogeorgos G, Sakas DE, Thalassinos NC. Tumor metastatic to the pituitary gland: case report and literature review. J Clin Endocrinol Metab 2004: 89: 574-580.

\section{Address for correspondence}

\section{Monika Łubińska}

Department of Endocrinology and Internal Diseases

University Clinical Centre

Medical University of Gdansk

Debinki 7

80-952 Gdansk

e-mail: mlubinska@gumed.edu.pl

Submitted: 13.02 .2012

Accepted: 8.05 .2013 